\title{
Tip leakage flow analysis of an axial turbine under the effect of separation at low Reynolds number
}

Proc IMechE Part A:

$J$ Power and Energy

$0(0) \mathrm{I}-15$

(C) IMechE 2019

Article reuse guidelines:

sagepub.com/journals-permissions

DOI: $10.1177 / 0957650919882877$

journals.sagepub.com/home/pia

(SSAGE

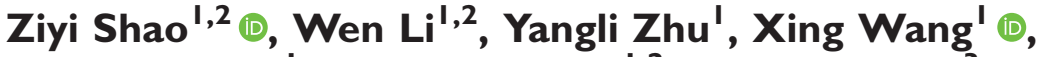 \\ Xuehui Zhang', Haisheng Chen ${ }^{1,2} \mathbb{\infty}$ and Wei Qin ${ }^{3}$
}

\begin{abstract}
The tip clearance flow could lead to work reduction and loss generation in turbomachines. However, the effect of separation at low Reynolds number on leakage flow is seldom studied. The previous method for evaluating tip leakage characteristics should also be further researched. Thus, numerical investigations on the tip clearance flow in an unshrouded axial-inflow turbine are conducted at low Reynolds number $\left(3.5 \times 10^{4}\right.$ of the rotor outlet at the designed condition) in the present study. The flow patterns and leakage mass flow rate of the clearance have been analyzed in detail. It is found that the tip clearance flow is greatly affected by the flow separation caused by low Reynolds number. The scraping ratio adopted in previous references does not accord with the clearance flow characteristics at low Reynolds number, especially in the front part of the clearance. A coefficient by -0.70 power of the Reynolds number is proposed to modify the scraping ratio in the present study. The synergy between the velocity and the pressure gradient is innovatively employed to research the tip clearance flow characteristics, and it gives a reliable criterion of indicating the flow patterns in the tip clearance.
\end{abstract}

\section{Keywords}

Turbines, turbomachinery flow, turbomachinery aerodynamics, turbine modeling

Date received: 21 May 2019; accepted: 18 September 2019

\section{Introduction}

It is widely considered that the tip clearance flow can significantly lead to work reduction and loss generation in turbomachinery. During the last three decades, there are a large volume of published studies focusing on the tip clearance flow. For example, detailed measurements were reported by Bindon, ${ }^{1}$ Moore and Tilton, ${ }^{2}$ Heyes and Hodson, ${ }^{3}$ and Yaras and Sjolander. ${ }^{4}$ The reverse flow of the separation bubble, which generates from the pressure side of the gap, was confirmed by smoke visualization over the tip gap. The mixing process of jet flow behind the separation bubble may cause a consequent increase in static pressure and entropy if the blade thickness is more than about four times the tip gap. ${ }^{5}$ Although the size of the clearance is usually about $1 \%$ of the blade height, the loss due to the leakage could not be neglected and the optimization on loss control has received considerable critical attention. The tip clearance loss in a turbine accounts for as much as one-third of the total loss. ${ }^{6,7}$ Some combined control technologies at the clearance region have been put forward recently, e.g. casing treatment and blowing, ${ }^{8}$ winglet and squealer, ${ }^{9,10}$ and honeycomb seal. ${ }^{11,12}$ However, the relative motion between the blade and shroud endwall has been neglected in these studies. The effect of relative motion had been investigated by Morphis and Bindon ${ }^{13}$ and Yaras and Sjolander. ${ }^{14}$ In their major studies, shear effects (or scraping flow) would presumably become more important at small clearances. The countereffect of the scraping flow reduces the leakage, whereas the flow pattern at the clearance has not been affected by it greatly. In another numerical study,

\footnotetext{
'Institute of Engineering Thermophysics, Chinese Academy of Sciences, Beijing, China

${ }^{2}$ School of Engineering Science, University of Chinese Academy of Sciences, Beijing, China

${ }^{3}$ Institute of Mechanics, Chinese Academy of Sciences, Beijing, China
}

\section{Corresponding author:}

Haisheng Chen, Institute of Engineering Thermophysics, Chinese Academy of Sciences, No.I I 4th Ring Road, Haidian, Beijing 100190, China.

Email: chen_hs@mail.etp.ac.cn 
Tallman and Lakshminarayana ${ }^{15}$ pointed out that losses associated with the leakage vortex are reduced due to the effect of scraping while the loss inside the gap increases. Still, the former advantage offsets the latter disadvantage.

Besides, analyses of the low Reynolds number flows involved in gas turbines are attracted more attention. In general, it is found by experiment that the effect of $R e$ on the performance of turbomachines is small when $R e$ is more than $2 \times 10^{5} .{ }^{16}$ The boundary layer on turbine blades is predominately turbulent and thin. It has little impact on the flow field in turbomachines. ${ }^{17}$ However, the flow field is further complicated at lower Reynolds number because the boundary layer could be laminar or undergoing transition to turbulence. The Reynolds number based upon the axial chord and inlet conditions could drop lower than $1 \times 10^{5} .^{18}$ Rizzetta and Visbal ${ }^{19}$ argued that low-pressure turbines of unmanned air vehicles (UAVs) could encounter Reynolds number below $2.5 \times 10^{4}$ because of a reduction in atmospheric density during the high-altitude cruise. Dorney et al. ${ }^{20}$ reported a serious of experiments and numerical studies on losses in the PAK-B low-pressure turbine whose Reynolds number are $4.3 \times 10^{4}, 8.6 \times 10^{4}$, and $1.72 \times 10^{5}$, respectively. Their results showed that the losses remain nearly constant between $\operatorname{Re}=8.6 \times 10^{4}$ and $R e=1.72 \times 10^{5}$. Nevertheless, the losses increase rapidly as the Reynolds number drops lower than $4.3 \times 10^{4}$ because there exists a mixture of separation bubble and natural transition on the suction surface. Similar phenomenon is also reported by Arakawa et al., ${ }^{21}$ and the passive surface modification is recommended to reduce this separation problem.

The current blade geometry is based on the axialflow turbine supplied by Dr Matsunuma. The unsteady flow characteristics and loss mechanism were investigated previously in the stator passage and rotor passage. ${ }^{18,22,23}$ So far, however, detailed measurements at the clearance region of turbine rotor have not been presented, especially in the flow structure and leakage mass flow rate. Unless the simulate modelling tests have been applied, ${ }^{24}$ it is difficult to measure and acquire credible aerodynamic parameters at such a narrow gap by conventional probes. There is a relative paucity of scientific literature specifically relating to the clearance flow characteristics at low Reynolds numbers recently. With the improvement of computational fluid dynamics (CFD) used to solve the Navier-Stokes equations, the numerical simulation could provide a detailed insight into the tip clearance flow. ${ }^{25,26}$ Thus, the researches on the tip clearance flow at low Reynolds numbers are conducted numerically in the current work. Firstly, the experimental validation and a brief overview of flow characteristics of rotor tip clearance are presented. In the following sections, the scraping ratio is employed and modified to study the scraping and leakage effects in the present axial turbine at low Reynolds numbers.
The synergy-based method is innovatively employed to research the clearance flow and the leakage mass flow rate in detail, and the validation has been done at off-design operating conditions.

\section{Research methodology}

\section{Experimental facility and conditions}

Detailed measurements of time-averaged and timedependent distributions of the aerodynamic parameters in the present axial-flow turbine were conducted by Dr Matsunuma. The turbine is an air suction type with a blower behind the turbine cascade. Inside and outside endwall diameters of the cascade are $350 \mathrm{~mm}$ and $500 \mathrm{~mm}$, respectively. The geometries and specifications of the nozzle and rotor cascades are shown in Figure 1(a) and Table 1. The flow conditions of stator inlet $(30 \mathrm{~mm}$ upstream from the stator leading edge at midspan), stator outlet ( $6.6 \mathrm{~mm}$ downstream from the stator trailing edge at midspan) and rotor domain were measured using a three-hole pressure probe, a five-hole pressure probe and a laser Doppler velocimetry (LDV) system, respectively.

Detailed aerodynamic parameters at designed operating condition are shown in Table 2. The Reynolds number at stator inlet was set to $R e_{\text {in,ST }}=2.0 \times 10^{4}$ based on the stator chord length. The Reynolds number at rotor outlet $R e_{\text {out,RT }}$ was calculated as $3.5 \times 10^{4}$ based on the rotor chord length from the measured LDV data. Previous researches ${ }^{17,27}$ have demonstrated that the effects of $R e$ on the performance of turbomachines is significant when $R e$ is less than $2.0 \times 10^{5}$. It is because at low $R e$, the viscous boundary layers on the blades of a turbomachine are generally laminar and very thick. Hence, the internal flow field at designed operating condition might be affected by the effect of low Reynolds number. The Mach numbers based on the massaveraged velocities at the stator inlet, stator outlet, rotor inlet, and rotor outlet were $M a_{\text {in,ST }}=0.013$, $M a_{\text {out }, \mathrm{ST}}=0.031, M a_{\text {in, RT }}=0.014$, and $M a_{\text {out }, \mathrm{RT}}=$ 0.027 , respectively. It should be noted that the flow at the designed operating condition should be treated as incompressible flow because all the Mach numbers are less than 0.3 .

\section{Numerical model}

The single-sector domains of the present axial-flow turbine contain four parts, including INBLOCK, STATOR, ROTOR, and OUTBLOCK. All the computational meshes are the structure hexahedral type with periodic boundaries, as presented in Figure 1(b). The grids of the INBLOCK, STATOR, and OUTBLOCK domains are generated by ANSYS TurboGrid automatically. The regions around the blade surface and the tip gap are discretized into O-type grids by ANSYS ICEM-CFD at the 


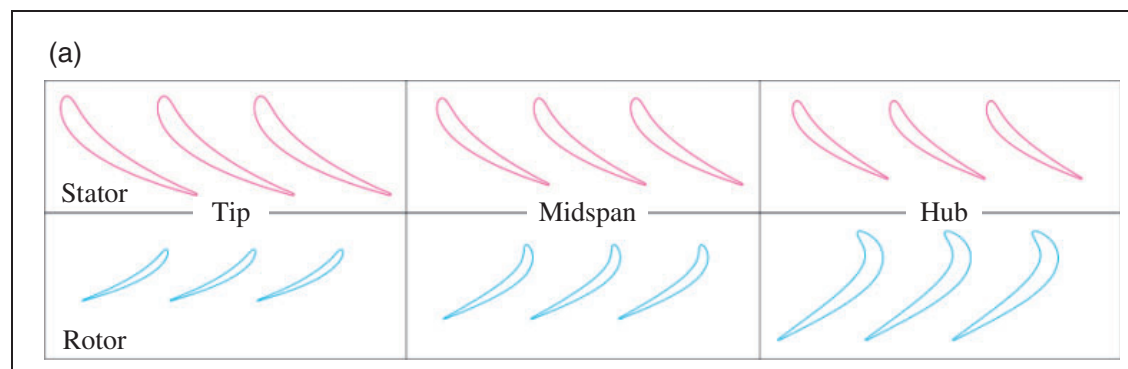

(b)

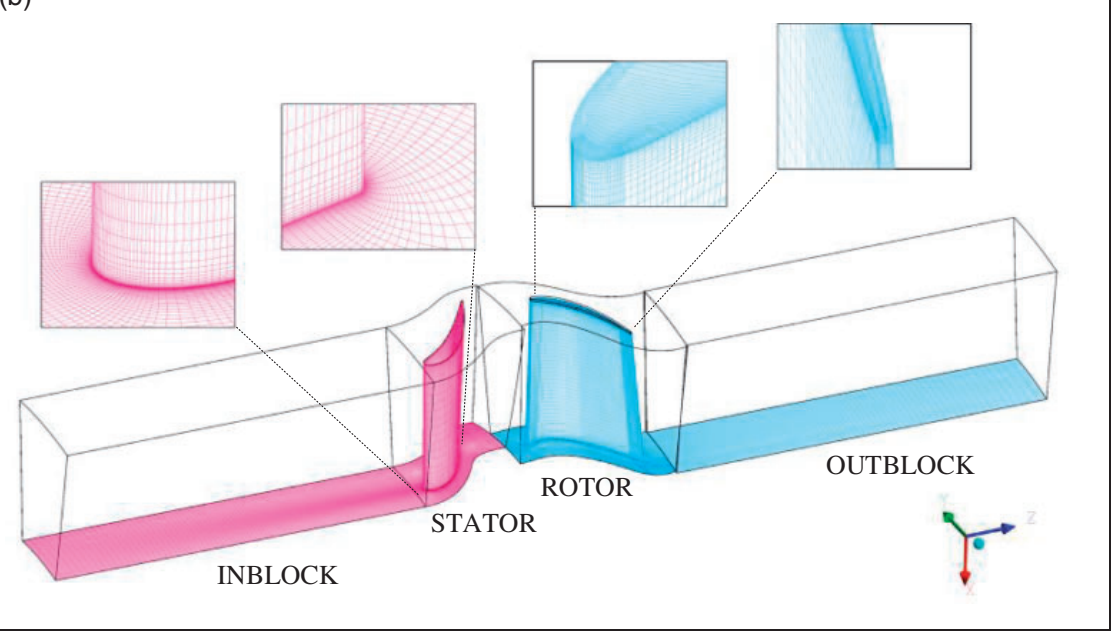

Figure I. Schematic of the axial-flow turbine: (a) blade geometry and (b) computational grids of single-sector domains.

Table I. Specifications of turbine cascades.

\begin{tabular}{lll}
\hline & Stator & Rotor \\
\hline Number of blades & 28 & 31 \\
Chord $(\mathrm{mm})$ & 67.6 & 57.5 \\
Pitch $(\mathrm{mm})$ & 47.7 & 43.1 \\
Blade height $(\mathrm{mm})$ & 75.0 & 74.0 \\
Inlet flow angle $\left(^{\circ}\right)$ & 0.0 & 22.1 \\
Outlet flow angle $\left(^{\circ}\right)$ & 67.4 & 63.4 \\
Stagger angle $\left({ }^{\circ}\right)$ & 51.0 & 44.8 \\
Tip clearance $(\mathrm{mm})$ & 0.0 & $1.0\left(\delta^{\prime}=1.35 \%\right)$ \\
\hline
\end{tabular}

ROTOR domain. The face angle of grids is between $15^{\circ}$ and $165^{\circ}$, and the maximum volume ratio and length ratio are less than 20 and 1,000, respectively. Hence, the current mesh quality is generally good and acceptable for turbomachinery simulation. ${ }^{28}$

The shear stress transport (SST) turbulence model $^{29}$ with gamma-theta transition model is adopted in the current steady simulation, and the equations of continuity, momentum and energy are solved by the high-resolution scheme in the commercial CFD solver ANSYS CFX. The connection method between stationary and rotating boundary (such as STATOR to ROTOR) is chosen as Stage, which performs a circumferential averaging of the fluxes through bands on the interface. The grid height in the viscous sublayer region near the wall is
Table 2. Boundary conditions at designed operating condition.

\begin{tabular}{llll}
\hline Domain & Component type & Parameter & Value \\
\hline INBLOCK & Stationary & $P t_{\text {in }}$ & $101,325 \mathrm{~Pa}$ \\
& & $T t_{\text {in }}$ & $293 \mathrm{~K}$ \\
& & $T u_{\text {in }}$ & $0.5 \%$ \\
STATOR & Stationary & - & - \\
ROTOR & Rotating & $\omega_{0}$ & $402 \mathrm{r} / \mathrm{min}$ \\
OUTBLOCK & Stationary & $P s_{\text {out }}$ & $101,195 \mathrm{~Pa}$ \\
\hline
\end{tabular}

fine enough ( $\max$ value $0.002 \mathrm{~mm}$ ) to ensure dimensionless wall distance $y^{+}<1$ so that the SST model gives a highly accurate prediction. Several off-design conditions are listed in Table 3. The rotating speed $\omega$ ranges from $322 \mathrm{r} / \mathrm{min}$ to $563 \mathrm{r} / \mathrm{min}$ (see VR1, VR2, and VR3), and the static pressure at outlet ranges from $101,295 \mathrm{~Pa}$ to $100,995 \mathrm{~Pa}$ (see VP1, VP2, and VP3). The SST turbulence model with gamma-theta transition model shows good convergence under designed and off-designed conditions. All the rootmean-square residuals for momentum and mass equations, energy equation, and turbulence equation are converged less than $1 \times 10^{-6}$. Grid independence studies are shown in Table 4, whose total nodes range from 290,000 to $4,440,000$. It can be pointed out that the difference of the total to static efficiency $\eta_{\mathrm{t}-\mathrm{s}}$ is less than $0.1 \%$ when the number of grids is beyond 
$3,370,000$. Thus, the following computational results are based on the case whose total grids are $3,370,000$.

\section{Analytical methods}

Seven cross-sectional planes, which are established equidistantly along the meridional position of the rotor blade tip $Z_{\mathrm{RT} \text {,tip, are employed to obtain local }}$ aerodynamic data, as illustrated in Figure 2. Each cross-sectional plane is normal to the main line (see the dotted line) of rotor blade tip. Besides, $\gamma$ is the angle between the tip main line and the meridional direction.

Some dimensionless coefficients are employed since it is easy to calculate them from cascade test data. ${ }^{5}$ The total pressure loss coefficient CPt, static pressure coefficient CPS, absolute velocity coefficient $V_{\text {abs }}$ and Reynolds number $R e$ are defined in the following way

$$
\begin{aligned}
& C P t=\frac{P t_{\text {in }}-P t}{P t_{\mathrm{out}}-P s_{\mathrm{out}}} \\
& C P s=\frac{P s-P s_{\mathrm{in}}}{P t_{\mathrm{out}}-P s_{\mathrm{out}}} \\
& V_{\mathrm{abs}}=\frac{V}{V_{\mathrm{ST}, \text { out }}} \\
& \operatorname{Re}=\frac{\rho u c}{\mu}
\end{aligned}
$$

\begin{tabular}{|c|c|c|c|}
\hline Conditions & VRI & VR2 & VR3 \\
\hline \multicolumn{4}{|c|}{ Variable rotating speed conditions: } \\
\hline$\omega(r / \min )$ & $322\left(80 \% \omega_{0}\right)$ & $482\left(120 \% \omega_{0}\right)$ & $563\left(140 \% \omega_{0}\right)$ \\
\hline$P s_{\text {out }, \text { OB }}(\mathrm{Pa})$ & & 101,195 & \\
\hline Conditions & VPI & VP2 & VP3 \\
\hline \multicolumn{4}{|c|}{ Variable pressure ratio conditions: } \\
\hline$P s_{\text {out, OB }}(\mathrm{Pa})$ & 101,295 & 101,095 & 100,995 \\
\hline$\omega(\mathrm{r} / \mathrm{min})$ & & $402\left(100 \% \omega_{0}\right)$ & \\
\hline
\end{tabular}

Table 3. Off-designed operating conditions. where $P t, P s$, and $V$ are total pressure, static pressure, and absolute velocity, respectively. The subscribe in, out and ST are the mass-averaged parameters at inlet plane, outlet plane, and STATOR domain. The Reynolds number is based on the blade chord length $c$, mass-averaged density $\rho$, mass-averaged velocity $u$ and mass-averaged dynamic viscosity $\mu$ at inlet or outlet surface.

In order to describe the scraping and leakage effects along the tip clearance, Dambach et al. ${ }^{24}$ put forward the scraping ratio $R$, which can be defined by the driving pressure difference and the scraping dynamic pressure

$$
R=\frac{\Delta P}{\frac{1}{2} \rho(U \cos \gamma)^{2}}
$$

\begin{tabular}{|c|c|c|c|c|c|c|c|}
\hline Grid no. & INBLOCK & STATOR & ROTOR & $\begin{array}{l}\text { TC } \\
\text { (in ROTOR) }\end{array}$ & OUTBLOCK & Total & $\eta_{\mathrm{t}-\mathrm{s}}(\%)$ \\
\hline I & 30,000 & 90,000 & 120,000 & 30,000 & 50,000 & 290,000 & 77.58 \\
\hline 2 & 50,000 & 250,000 & 400,000 & 100,000 & 200,000 & 900,000 & 77.98 \\
\hline 3 & 100,000 & 800,000 & $\mathrm{I}, 000,000$ & 250,000 & 300,000 & $2,200,000$ & 78.44 \\
\hline 4 & 300,000 & $1,200,000$ & $\mathrm{I}, 500,000$ & 350,000 & 370,000 & $3,370,000$ & 78.46 \\
\hline 5 & 350,000 & $1,500,000$ & $2,100,000$ & 500,000 & 450,000 & $4,400,000$ & 78.43 \\
\hline
\end{tabular}

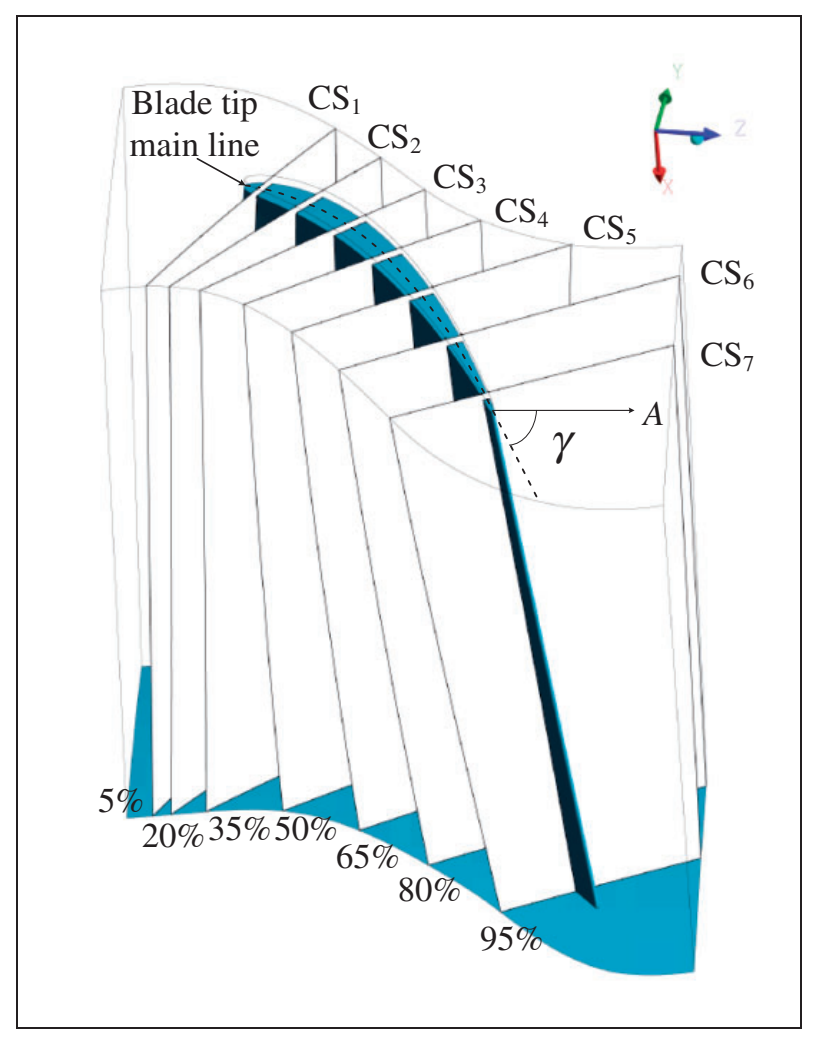

Figure 2. Schematic of cross-sectional planes in the rotor tip clearance region.

Table 4. Grid independence. 
where $\Delta P$ is the driving pressure difference across the tip gap, from the pressure side to suction side, and $1 / 2 \rho(U \cos \gamma)^{2}$ is the scraping dynamic pressure. The scraping ratio was initially applied to the radialinflow turbine. The scraping dynamic pressure is mainly related to the blade circumferential velocity $U$ because of the variation of the radial distance. Although the $U$ of rotor blades keeps almost constant in an axial turbine, the $\gamma$ related to the local blade angle dominates the scraping dynamic pressure. Thus, the scraping ratio could be applied to the axial turbine.

Yaras and Sjolander ${ }^{14}$ argued that the flow structures in the clearance were dominated by two possible mechanisms: the shear effect (the negative region) and the leakage effect (the positive region), as shown in Figure 3. This model was also employed in the backface clearance in a radial turbine rotor, ${ }^{26}$ and Serrano et al. ${ }^{30}$ presented the expressions of the positive $m_{+}$ (see equation (6)) and negative $m_{-}$(see equation (7)) tip leakage flow. The net leakage mass flow $m_{l}$ has been proposed along the suction side with the definition of equation (8)

$$
\begin{aligned}
\frac{m_{+}}{G} & =\frac{\int_{A_{+}} \rho \bar{w}_{\theta+} \mathrm{d} A_{+}}{G} \\
\frac{m_{-}}{G} & =\frac{\int_{A_{-}} \rho \bar{w}_{\theta-} \mathrm{d} A_{-}}{G} \\
\frac{m_{l}}{G} & =\frac{m_{+}}{G}+\frac{m_{-}}{G}
\end{aligned}
$$

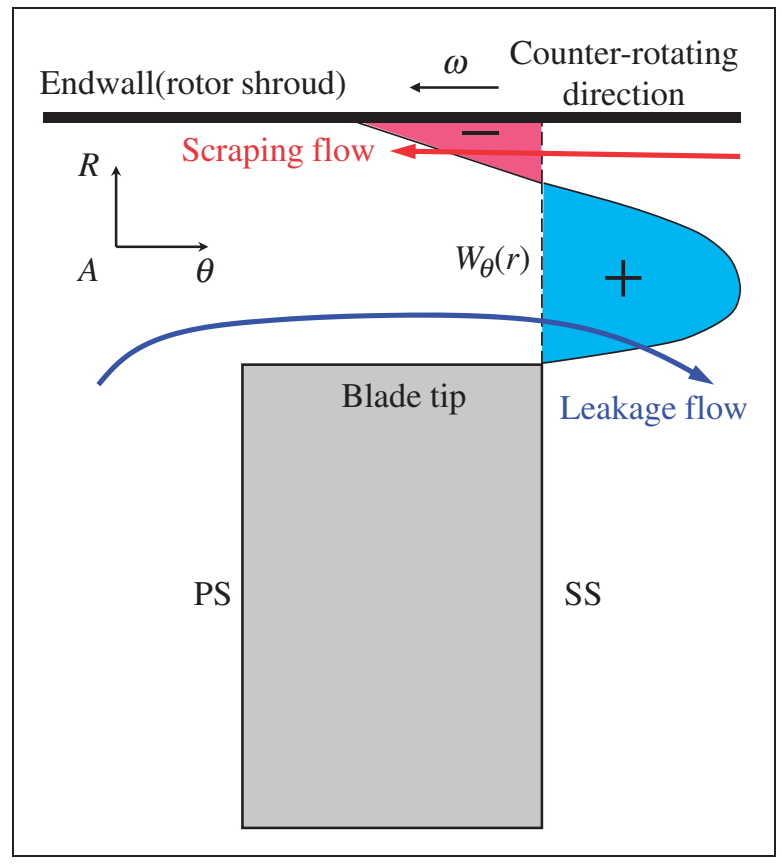

Figure 3. Tip clearance flow characteristics in rotating reference frame. where $w, A$, and $G$ are the relative velocity, area and turbine mass flow rate, respectively. The subscribe + , - , and $\theta$ are the positive region, negative region, and circumferential component, respectively. However, little researches have focused on identifying and measuring the boundary of the two regions at such a narrow gap, and it is difficult to calculate the leakage mass flow rate by equation (8). Further researches are required to explore a post-processing method for calculating the leakage mass flow rate, which could distinguish $A_{+}$and $A_{-}$credibly. On the basis research of physical quantity synergy, Liu et al. ${ }^{31,32}$ proposed the synergy angle $\beta$, which presents the relationship between the velocity vector and pressure gradient. In a rotating coordinate system of the turbine rotor, it can be expressed as

$$
\beta=\arccos \left(\frac{\boldsymbol{w} \cdot \nabla p}{|\boldsymbol{w}| \cdot|\nabla p|}\right)
$$

Equation (9) shows the synergy relation between the relative velocity $\boldsymbol{w}$ and the pressure gradient $\nabla p$, and the synergy angle at the suction side of clearance is displayed in Figure 4. The pressure gradient (see green arrows), whose direction is from the pressure side to suction side, is normal to the suction surface. The synergy angle of the positive region (see blue arrows) is less than $90^{\circ}$ while it is more than $90^{\circ}$ in the negative region (see red arrows). Hence, the synergy angle, whose critical value is $90^{\circ}$, shows advantages of researching the shear and leakage effect of tip clearance. The distribution of synergy angle and

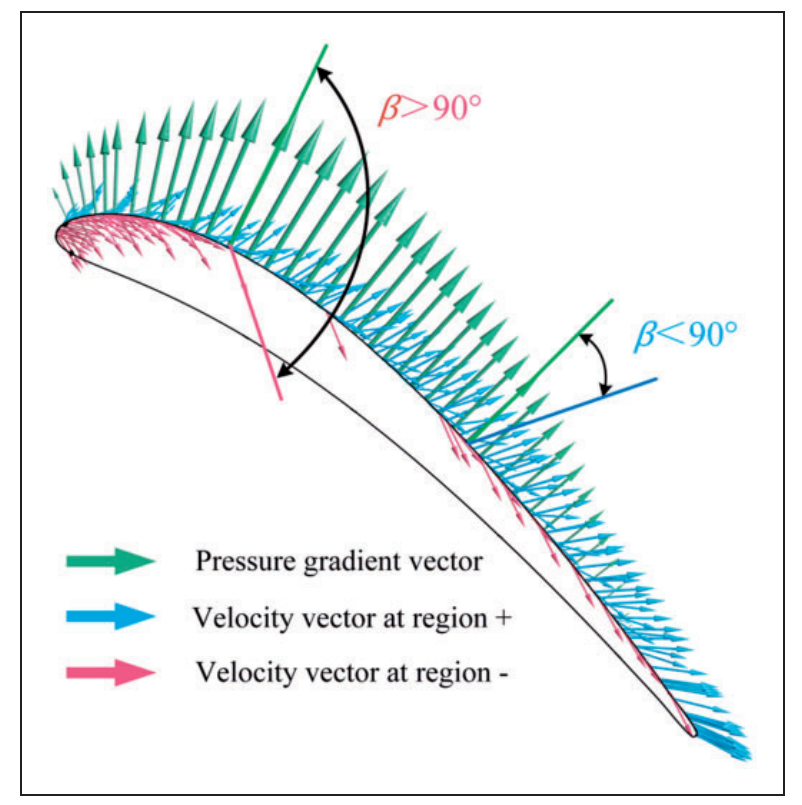

Figure 4. Schematic of the vector distribution and synergy angle at suction side of tip clearance. 
synergy-based leakage mass flow rate will be discussed in a later section in detail.

\section{Results and discussion}

\section{Experimental validation}

Both the experimental and computational results of the designed condition are compared in Table 5. The maximum relative deviation is less than $5.0 \%$. To validate the reliability of present simulations in detail, comparisons between the experimental and numerical results are made at stator inlet, stator blade, stator outlet, and rotor outlet, respectively.

Stator inlet conditions. Figure 5 shows the spanwise distributions of $C P t$ and $V / V_{\text {in,ST }}$ at the stator inlet plane. It could be concluded that the loss and velocity gradient are small, and the main flow is uniform. The inlet boundary layers at the designed condition are also validated in Table 6. Schlichting and Gersten ${ }^{33}$ pointed out that the shape factor of $H_{12}=2.59$ in the laminar region decreases to $H_{12}=1.4$ in the turbulent region. As can be seen in Table 6, the shape factors of the experiment and present simulation are closer to

Table 5. Overall performance comparisons for the whole turbine stage.

\begin{tabular}{llll}
\hline Parameter & EXP result & CFD result & Deviation (\%) \\
\hline$G\left(\mathrm{~kg} \mathrm{~s}^{-1}\right)$ & 0.537 & 0.536 & -0.2 \\
$R \mathrm{e}_{\text {in,ST }}$ & 20,000 & 19,858 & -0.8 \\
$M a_{\text {in,ST }}$ & 0.0130 & 0.0131 & +0.8 \\
$M a_{\text {out }, \mathrm{ST}}$ & 0.0310 & 0.0318 & +3.2 \\
$M a_{\text {in,RT }}$ & 0.0140 & 0.0146 & +4.3 \\
$M a_{\text {out }, \mathrm{RT}}$ & 0.0270 & 0.0280 & +3.6 \\
$R e_{\text {out }, \mathrm{RT}}$ & 35,000 & 35,973 & +2.8 \\
\hline
\end{tabular}

EXP: experimental; CFD: computational fluid dynamics.

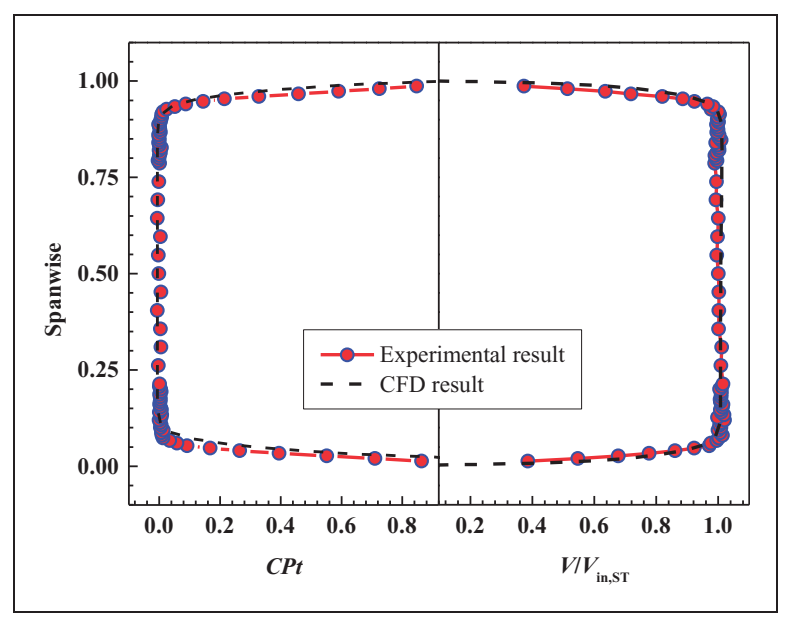

Figure 5. Performance comparison between the experimental and numerical results at stator inlet. the laminar regime, and the present inlet conditions of the numerical simulation are reliable.

Flow patterns on the stator blade. Figure 6 shows the flow patterns at the stator suction surface. The streamlines of the numerical result agree well with the oil-film visualization. ${ }^{22}$ There is a separation line of the laminar boundary layer on the blade surface due to the low Reynolds number flow. In addition, a reverse flow region is observed from the blade tip to the hub endwall. It conflicts with the passage vortex near the hub endwall.

Stator outlet conditions. Figure 7 shows the contour of $V_{\text {abs }}$ on the stator outlet plane. Both the experimen$\operatorname{tal}^{22}$ and numerical results show that the wake and secondary vortexes generate low-velocity regions near the trailing edge of stator blade. Matsunuma ${ }^{22}$ claimed that the passage vortexes sweep up the boundary layer on the stator surface, which cause the velocity deficit near the tip and hub endwalls.

Rotor outlet conditions. Figure 8 shows the aerodynamic parameters at the exit of rotor. The maximum relative deviation of $\alpha_{\text {out,RT }}$ and $V_{\mathrm{abs}}$ is $10.03 \%$ and $8.53 \%$, respectively. The CFD results agree well with the experimental data, at the tip clearance in particular. Taken together, these results indicate that the current numerical method and data are credible.

\section{Flow characteristics of rotor tip clearance}

For a better understanding of the detailed flow patterns in the tip clearance, streamlines coupled with CPS contour are displayed in Figure 9. Several typical flow patterns appear in the clearance, including the tip leakage flow, scraping vortex (SV) and separation bubble (SB1). It could be concluded that the tip clearance flow has been greatly affected by the flow separation at low Reynolds conditions generally. There exists a separation bubble (SBE) near the endwall casing, and it ranges from $20 \% Z_{\mathrm{RT} \text {,tip }}$ to $35 \% Z_{\mathrm{RT} \text {,tip }}$ approximately, as shown in Figure 9(b) and (c). That is because the boundary layer is relatively thick, and it is more susceptible to flow separation at low Reynolds number. Besides, a local low-pressure region could be seen from Figure 9(a), which attaches to the rotor suction side. The local low pressure may cause the

Table 6. Comparisons of endwall boundary layers at the stator inlet plane.

\begin{tabular}{lllllll}
\hline & \multicolumn{2}{ll}{ EXP $^{22}$} & & & \multicolumn{2}{l}{ CFD } \\
\cline { 2 - 3 } \cline { 6 - 7 } & Shroud & Hub & & Shroud & Hub \\
\hline Displacement thickness $\delta_{1}$ & 1.85 & 1.68 & & 1.54 & 1.45 \\
Momentum thickness $\delta_{2}$ & 0.832 & 0.730 & & 0.643 & 0.578 \\
Shape factor $H_{12}$ & 2.22 & 2.30 & & 2.40 & 2.51 \\
\hline
\end{tabular}

EXP: experimental; CFD: computational fluid dynamics. 


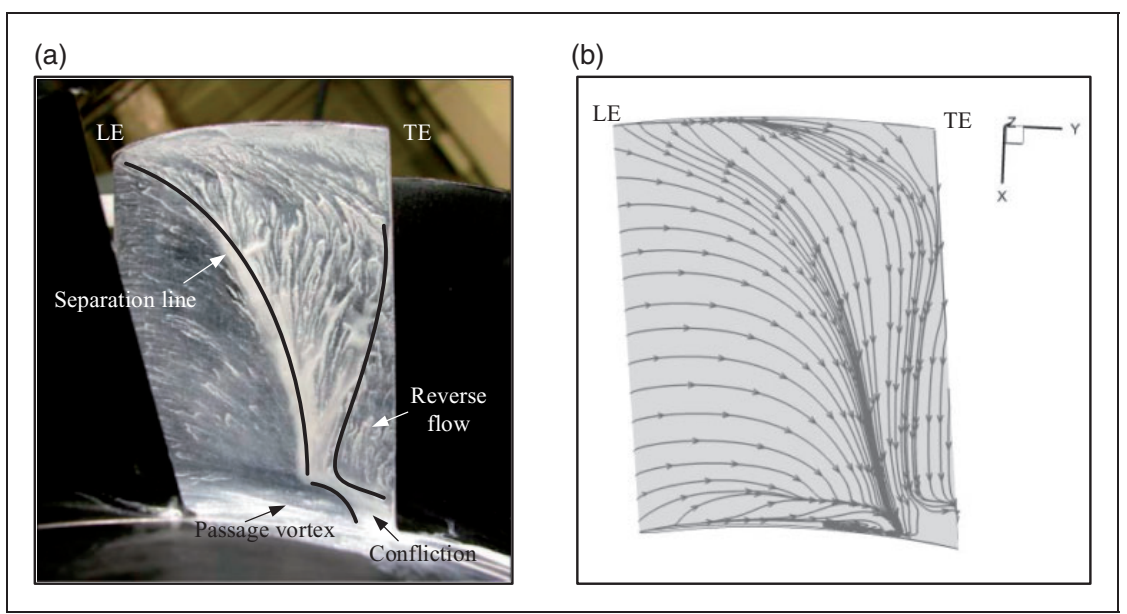

Figure 6. Schematic of flow patterns at nozzle suction surface: (a) EXP result (oil-film visualization) ${ }^{22}$ and (b) CFD result.

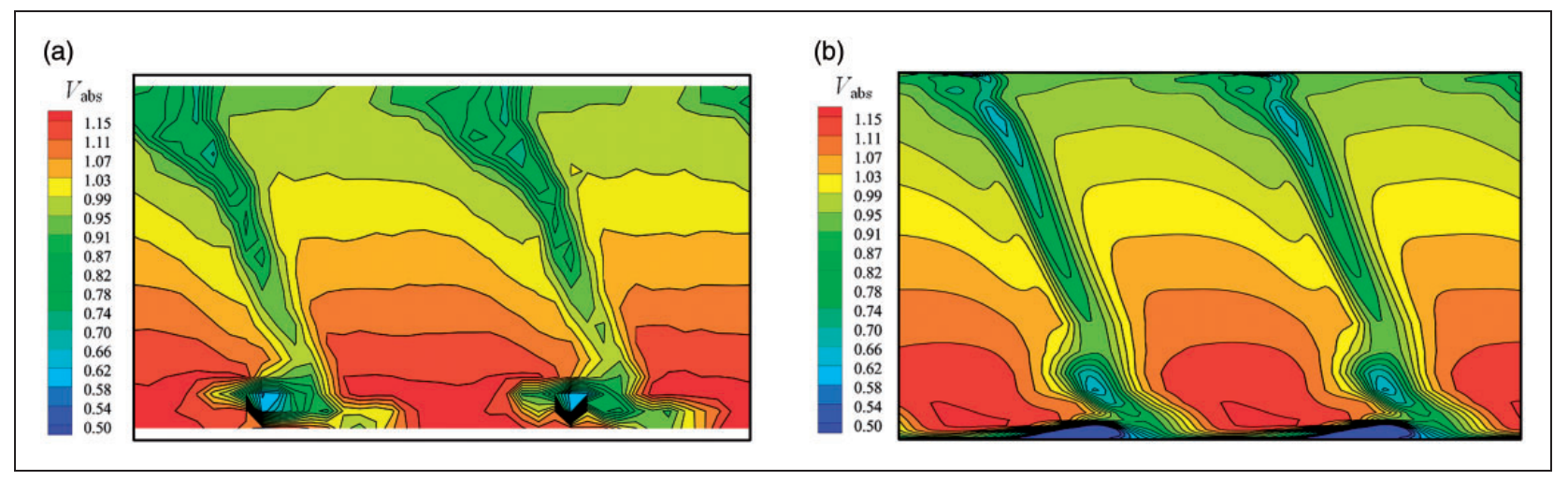

Figure 7. Comparisons of $V_{a b s}$ between the (a) experimental ${ }^{22}$ and (b) numerical results at stator outlet.

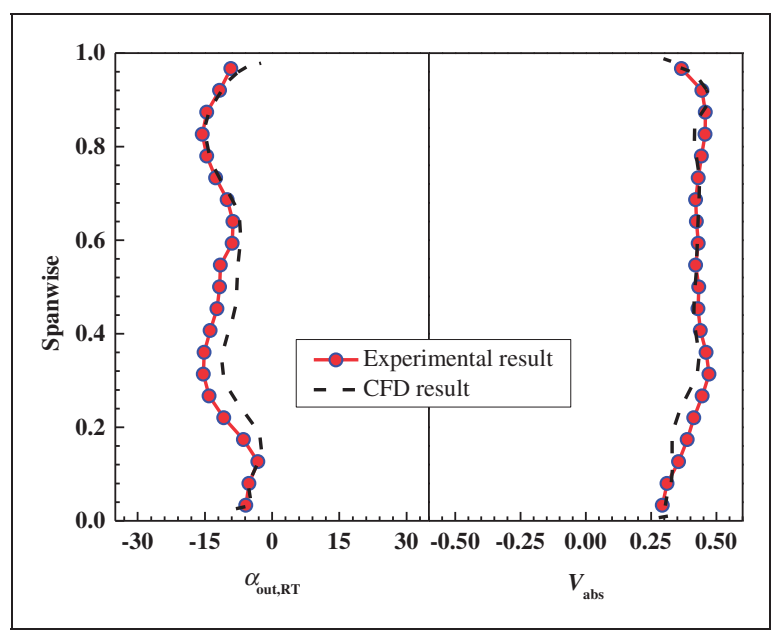

Figure 8. Performance comparison between the experimental and numerical results at rotor outlet.

diffusion of boundary layer and generate other separation regions, as indicated from Figure 9(b) to (f). Finally, the flow separation develops into an open separation bubble (SB2) at blade tip near the trailing edge, as shown in Figure 9(g). Although previous studies $^{34,35}$ have researched the flow separation on the blade suction surface, the open separation bubble on the blade tip, which comes from the flow separation, remains poorly discussed.

Comparisons of wall pressure and flow patterns among cross-sectional planes are shown in Figure 10. Distributions of CPS and flow patterns are similar from $5 \% Z_{\mathrm{RT} \text {,tip }}$ to $65 \% Z_{\mathrm{RT} \text {,tip. So, only }}$ the results at CS1 and CS5 are shown. In Figure 10(a) and (b), the reattachment line of SB1 appears almost near the place where the tip pressure is roughly equal to the endwall one. Similarly, the relationship between the reattachment line and static pressure was also confirmed by Sjolander and $\mathrm{CaO}^{36}$ in a linear turbine cascade. Figure 10(c) shows that the tip pressure reaches a peak while it is still lower than that at endwall, the SB1 occupies blade tip surface entirely and the reattachment line disappears. At $95 \% Z_{\mathrm{RT} \text {,tip, the }}$ SB2 occurs when the static pressure on suction side is higher than that on the pressure side, as shown in Figure 10(d). As the pressure difference between the pressure side and suction side decreases, the separation region expands and moves upward. It replaces the SB1 on the blade tip eventually when the static pressure on suction side is higher than that on the pressure side, as displayed in Figure 10(d). In summary, the tip clearance flow has been greatly affected 


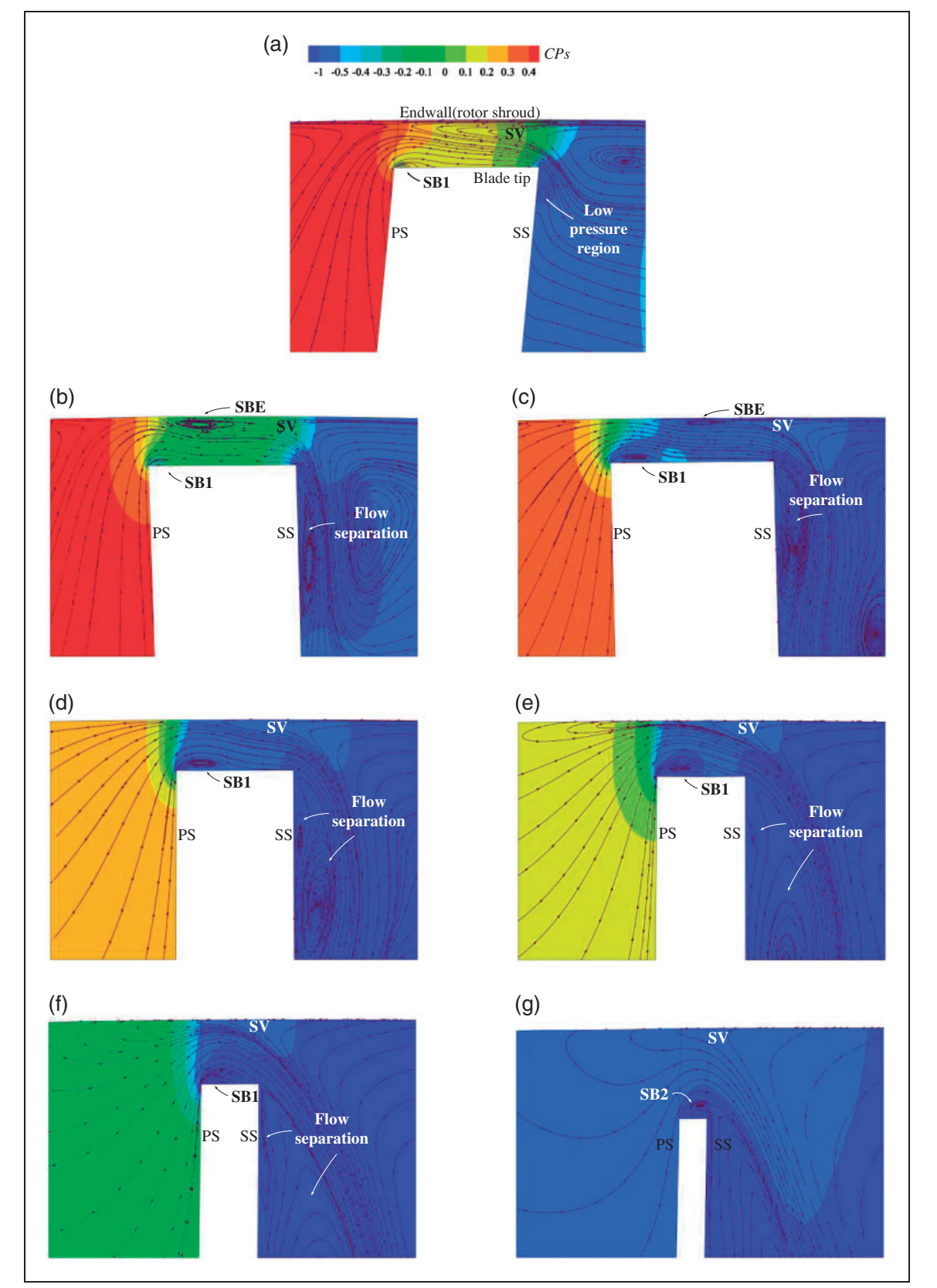

Figure 9. Streamlines on the cross-sectional plane: (a) $\mathrm{CS}_{1}$, (b) $\mathrm{CS}_{2}$, (c) $\mathrm{CS}_{3}$, (d) $\mathrm{CS}_{4}$, (e) $\mathrm{CS}_{5}$, (f) $\mathrm{CS}_{6}$, and (g) $\mathrm{CS}_{7}$. SV: scarping vortex; SB: separation bubble; PS: pressure side; SS: suction side.

by the flow separation in a low-Re environment, and the development of flow separation appears to be related to the low-pressure region on the blade suction surface.

\section{Scraping ratio model and modification}

Although the scraping ratio model has been proposed $^{24}$ and applied in the radial turbine, ${ }^{37}$ it is still necessary to validate its reliability in the present axial turbine. The scraping ratio and velocity vectors in the clearance are displayed in Figure 11(a), which is under the condition of $P s_{\text {out }}=99,695 \mathrm{~Pa}$ and $\omega=1,608 \mathrm{r} / \mathrm{min}$.
The Reynolds number $\left(R e_{\text {out, RT }}=1.3 \times 10^{5}\right)$ is relatively higher than that of the designed condition $\left(R e_{\text {out } \mathrm{RT}}=3.6 \times 10^{4}\right)$. It could be concluded that the scraping ratio agrees well with the flow pattern. The scraping effect dominates the flow in $\mathrm{CS}_{1}$, where the $R$ is less than 1. The leakage flow appears from $\mathrm{CS}_{2}$ to $\mathrm{CS}_{7}$ and the $R$ is more than 1. The results of the designed condition are illustrated in Figure 11(b). Figure 11(b) shows that the $R$ is less than 1 while there still exists leakage flow at $\mathrm{CS}_{1}$ (see Figure 9a). It could be seen from Figure 12(a) that the driving pressure difference $\Delta P$ increases rapidly from the leading edge to $\mathrm{CS}_{1}$. Such a sharp increase of pressure 


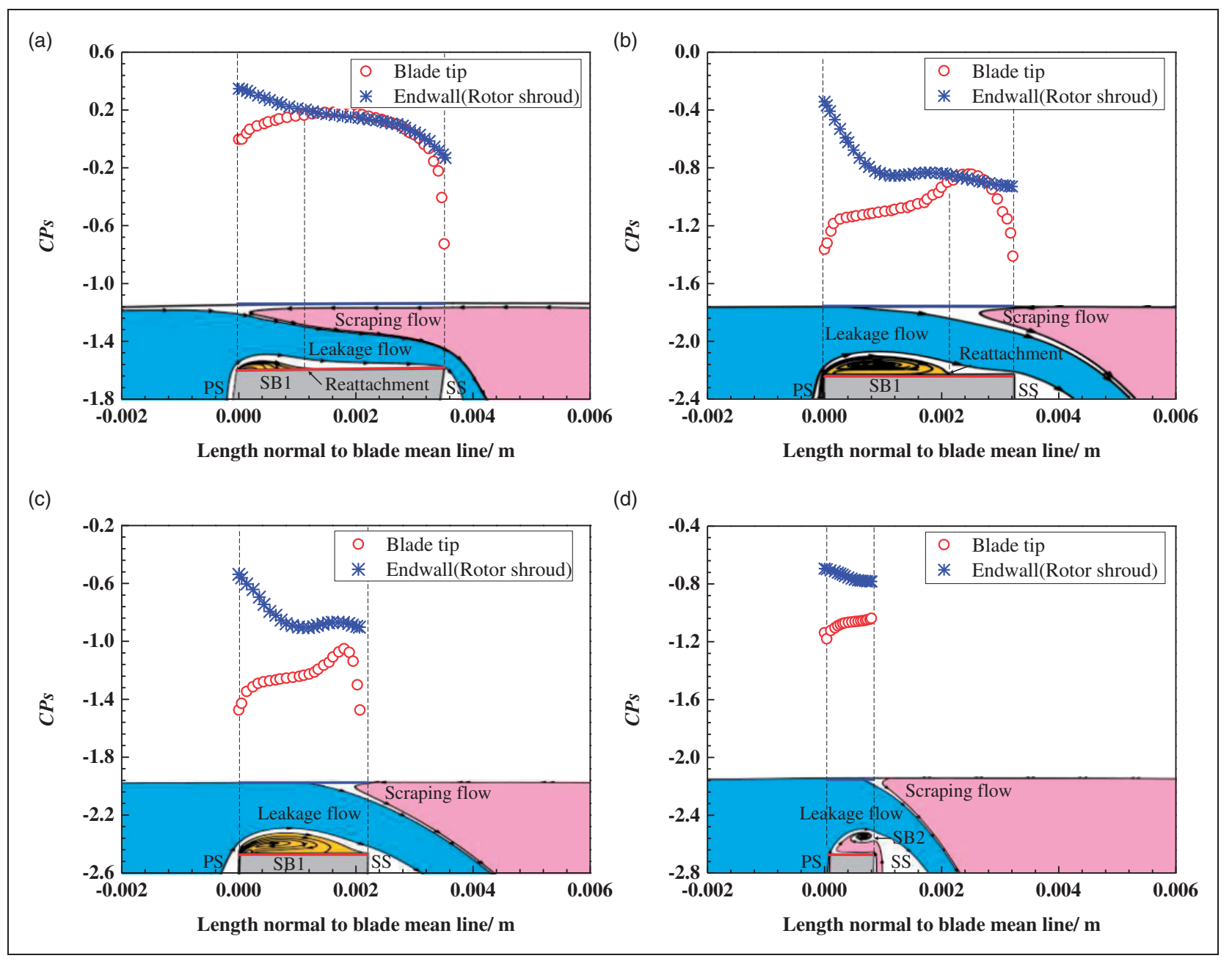

Figure 10. Relationships between wall pressure and flow patterns among cross-sectional planes: (a) $\mathrm{CS}_{1}$, (b) $\mathrm{CS}_{5}$, (c) $\mathrm{CS}_{6}$, and (d) $\mathrm{CS}_{7}$.
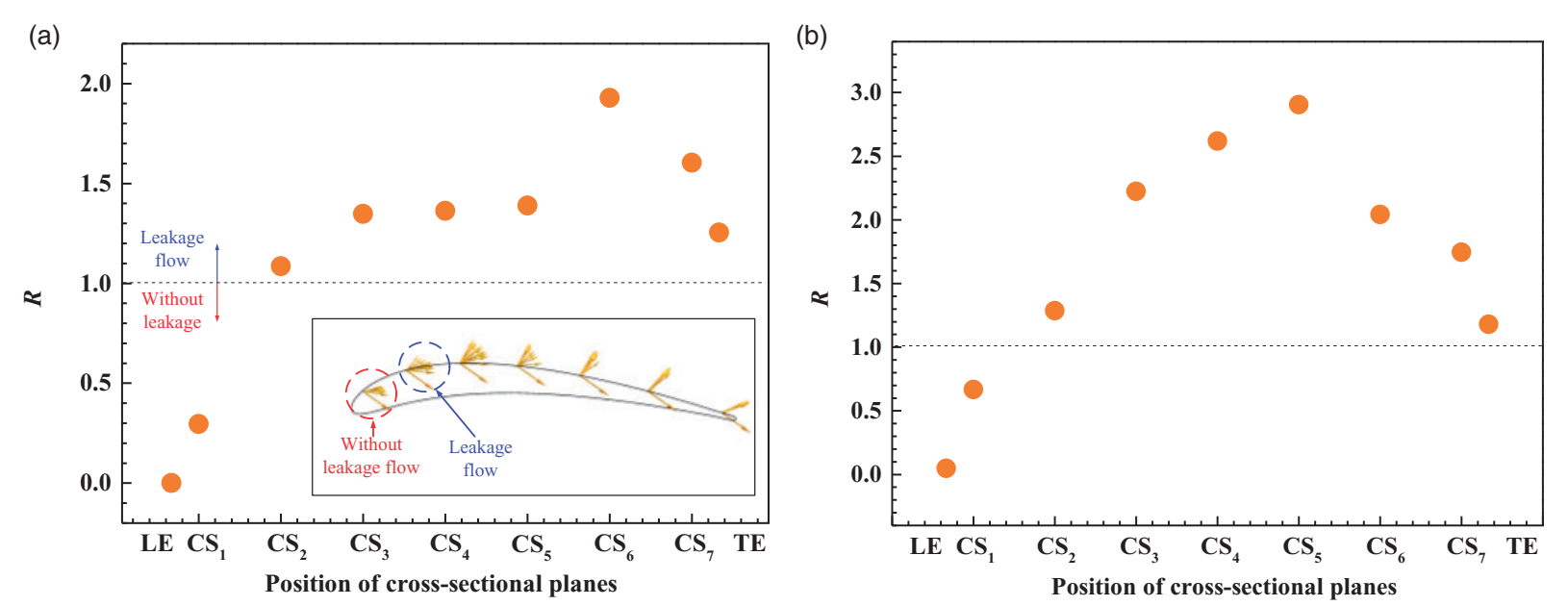

Figure II. Distributions of scraping ratio along the flow direction at: (a) $R e_{\text {out }, R T}=1.3 \times 10^{5}$ and (b) $\operatorname{Re}_{\text {out }, R T}=3.6 \times 10^{4}$. 


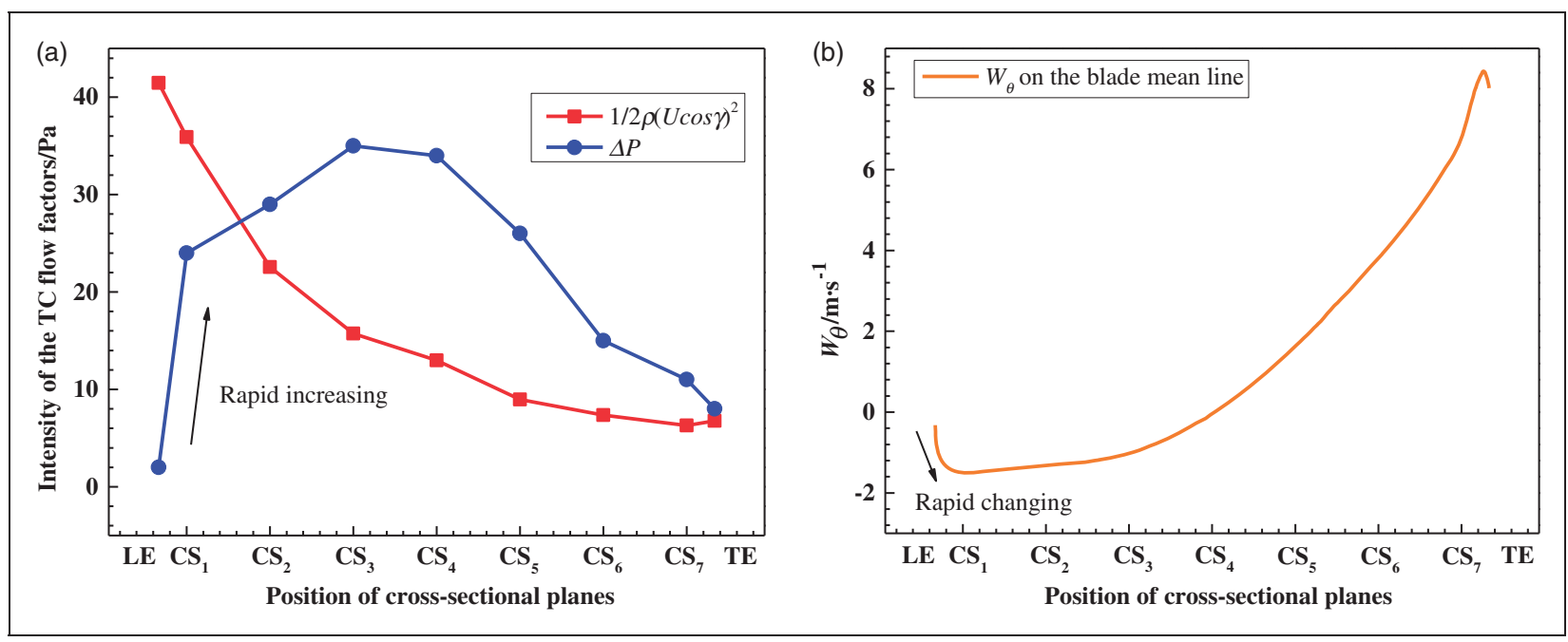

Figure 12. (a) Intensity of scraping and leakage flow and (b) circumferential velocity along the flow direction at designed condition.

difference contributes to the development of local inertial force at the clearance region, which might cause the leakage flow even if $\Delta P$ is less than $1 / 2 \rho(U \cos \gamma)^{2}$. It is assumed that aerodynamic parameters on the cross-sectional planes could be analyzed by the $\theta$-component. A possible explanation for the increase of leakage pressure could be concluded from the $\theta$-component of momentum equation in a rotating cylindrical coordinate frame

$$
\begin{aligned}
& \rho\left(\frac{\partial w_{\theta}}{\partial t}+w_{r} \frac{\partial w_{\theta}}{\partial r}+\frac{w_{\theta}}{r} \frac{\partial w_{\theta}}{\partial \theta}+\frac{w_{r} w_{\theta}}{r}+w_{z} \frac{\partial w_{\theta}}{\partial z}\right) \\
& =-\frac{1}{r} \frac{\partial p}{\partial \theta}+\rho g_{\theta}-2 \rho \omega w_{r} \\
& \quad+\mu\left[\frac{1}{r} \frac{\partial}{\partial r}\left(r \frac{\partial w_{\theta}}{\partial r}\right)-\frac{w_{\theta}}{r^{2}}+\frac{1}{r^{2}} \frac{\partial^{2} w_{\theta}}{\partial r^{2}}+\frac{2}{r^{2}} \frac{\partial w_{r}}{\partial \theta}+\frac{\partial^{2} w_{\theta}}{\partial z^{2}}\right]
\end{aligned}
$$

According to the observation done by Yaras et al., ${ }^{38}$ the chordwise momentum in the tip clearance could be seen as conserved. The radial component of relative velocity $w_{r}$ and the Coriolis force $-2 \omega w_{r}$ at the gap have been neglected. It is also assumed that the relative velocity remains almost unchanged in the tangential and axial direction. Therefore, equation (10) in a steady state could be expressed as

$$
\frac{1}{\mu} \frac{\partial p_{P S-S S}}{\partial \theta}=\frac{\partial}{\partial r}\left(r \frac{\partial w_{t i p, \theta}}{\partial r}\right)-\frac{w_{t i p, \theta}}{r}
$$

From equation (11), the pressure gradient through the gap is related to the circumferential component of relative velocity $w_{\theta}$. Figure $12(\mathrm{~b})$ presents the $w_{\theta}$ at tip main line (see the dotted line in Figure 2) from simulation results. The $w_{\theta}$ is about 0 at the leading edge of the clearance inlet. Hence, the pressure gradient here equals 0 . Because of the circumferential disturbance of the blade rotating, the $w_{\theta}$ changes rapidly from the

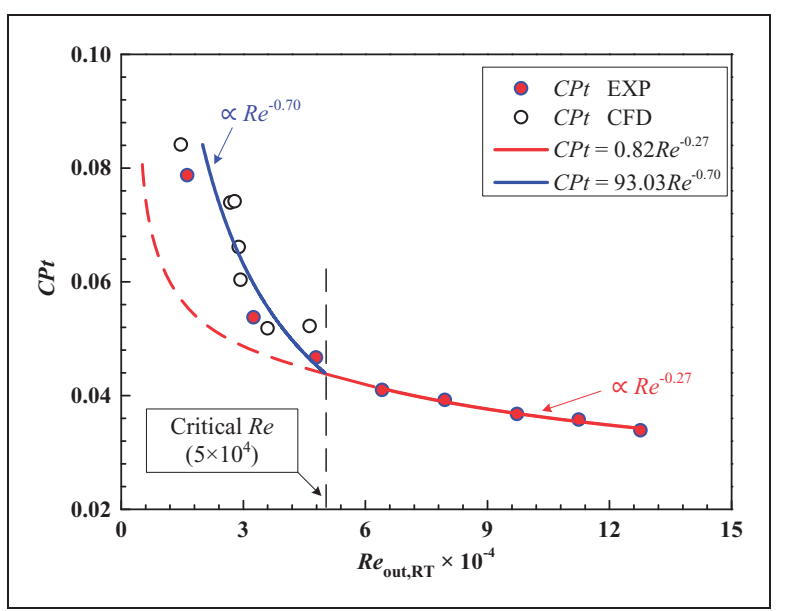

Figure 13. Total pressure loss under off-design operating conditions $\left(\delta^{\prime}=1.35 \%\right)$.

leading edge to $\mathrm{CS}_{1}$, and the pressure gradient also presents a sharp increase in such a short distance. Taken together, these analyses above show that the distribution of scraping ratio at low Reynolds number condition is quite different from previous researches. ${ }^{24,37}$

Before proceeding to modify the scraping ratio, it is necessary to investigate the effects of Reynolds number on the present axial turbine, as indicated in Figure 13. In the loss analyses proposed by Ainley and Mathieson, ${ }^{27}$ the performance of tip loss roughly obeys the -0.2 power of the Reynolds number around a mean Reynolds number of $2 \times 10^{5}$. At lower Reynolds number $\left(5 \times 10^{4}\right)$, it is anticipated that the efficiency may decrease rather more rapidly. The experimental $^{22}$ and numerical results are shown as filled circles and hollow circles, respectively. At higher Reynolds number $\left(R e_{\text {out.RT }}>5 \times 10^{4}\right)$, the results agree with about -0.27 power of the Reynolds number, as presented by the red curve in the figure. A more significant increase of total 
pressure loss is recorded among the experimental and numerical data when $R e_{\text {out, RT }}$ is less than $5 \times 10^{4}$. The blue curve of the -0.70 power of the Reynolds number fits closer than the red curve. The results of tip loss are in agreement with Ainley's findings in the present axial turbine approximately. Hence, a modified scraping ratio $R_{\mathrm{m}}$ is set up, which could be expressed as equation (12) in the following

$$
R_{\mathrm{m}}=R \cdot\left(\frac{R e_{\mathrm{out}, \mathrm{RT}}}{5 \times 10^{4}}\right)^{\psi}
$$

where the critical Reynolds number of $5 \times 10^{4}$ is chosen for fitting, and the $\psi$ is the coefficient, which is -0.70 in the present study. As analyzed above, the $\psi$ should be less than -0.2 because of the fall in performance at lower Reynolds numbers.

From Figures 14 and 15, velocity vectors are shown to indicate the flow characteristics at the suction side of tip clearance and validate the modified results. The circumferential velocity $U$ of the blade increases at higher rotating speed. Thus, the intensity of scraping flow $1 / 2 \rho(U \cos \gamma)^{2}$ is enhanced while the leakage flow becomes weaker. Figure 14(a) shows the leakage flow (see red dotted circles) occurs initially on the $\mathrm{CS}_{1}, \mathrm{CS}_{1}, \mathrm{CS}_{2}$, and $\mathrm{CS}_{2}$ at VR1, DC, VR2, and VR3 condition, respectively. The $R_{\mathrm{m}}$ shows a superior level compared to $R$, and it agrees well with the flow patterns under all variable rotating speed conditions, as shown in Figure 14(b). When pressure ratio rises, the intensity of leakage flow $\Delta P$ increases while the scraping flow becomes weaker. Figure 15(a) shows the leakage flow (see red dotted circles) occurs initially on the $\mathrm{CS}_{3}, \mathrm{CS}_{1}$, $\mathrm{CS}_{1}$, and $\mathrm{CS}_{1}$ at VP1, DC, VP2, and VP3 condition, respectively. It could be seen that the scraping ratio modified by Reynolds number also performs well under variable pressure ratio conditions, as shown in Figure 15(b). In addition, it should be point out that the coefficient $\psi$ can be varied with different turbine blades operated at other low Reynolds number conditions while the form of the equation does not need to be adjusted. The coefficient should be redefined by the CPt-Re curve in Figure 13. The method for modification may be more valuable than a quantitative coefficient.

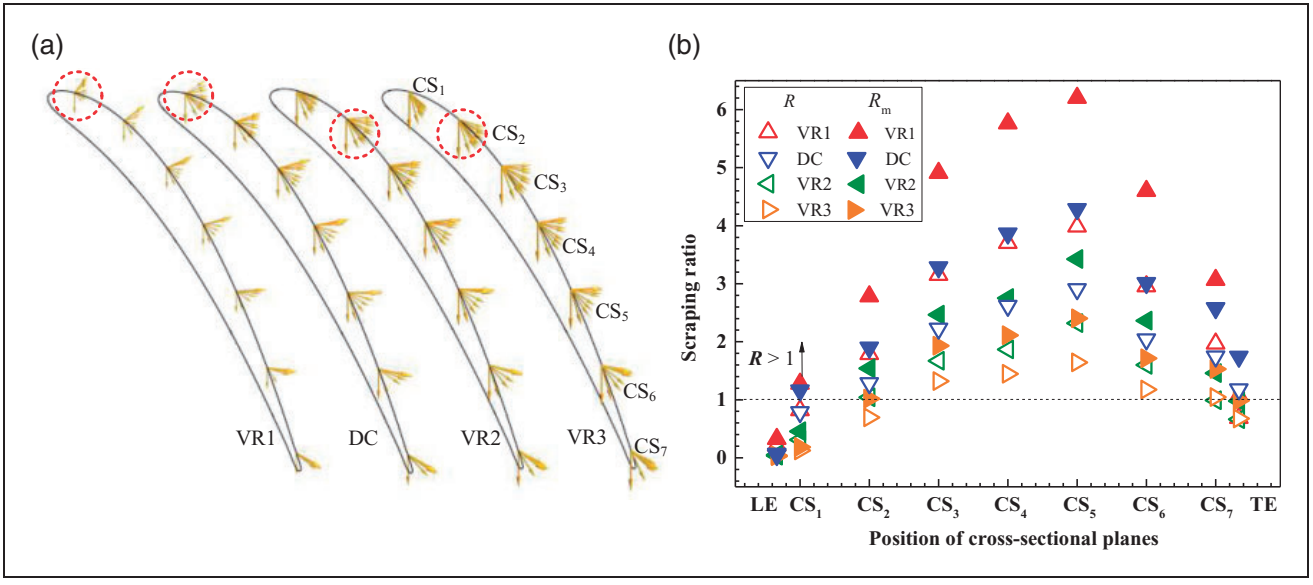

Figure 14. Tip clearance flow characteristics under various rotating speed conditions: (a) velocity vectors and (b) $R$.

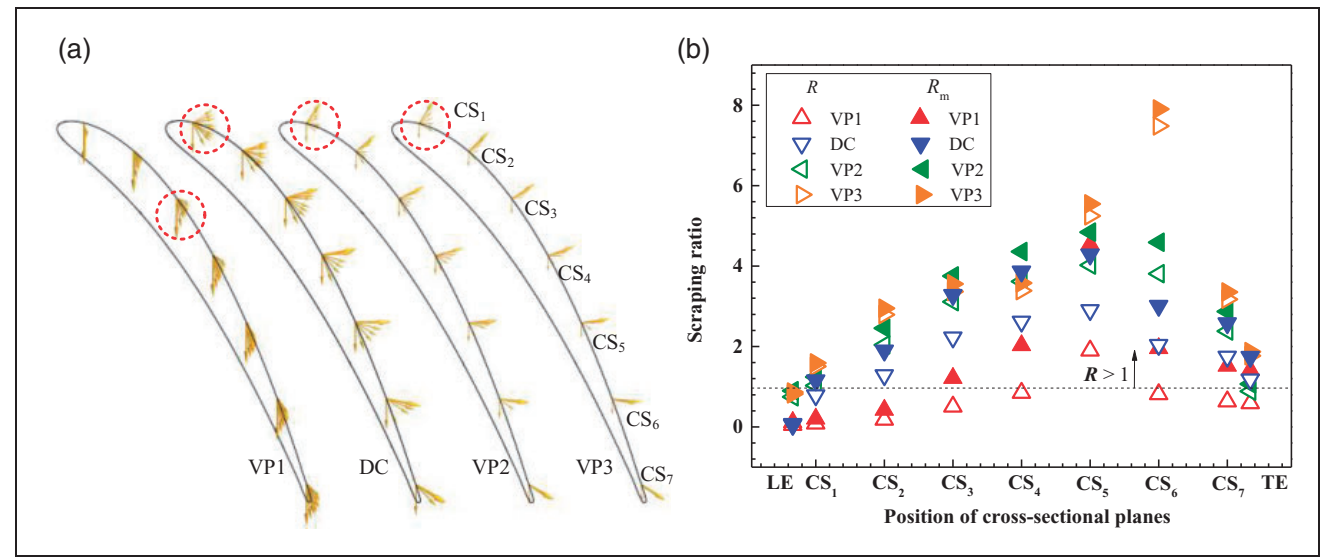

Figure 15. Tip clearance flow characteristics under various pressure ratio conditions: (a) velocity vectors and (b) $R$. 


\section{A synergy-based criterion and mass flow calculation}

As analyzed in an earlier section, the synergy method demonstrates the advantage of indicating flow patterns in the clearance region. The result of the synergy angle $\beta$ is displayed in Figure 16. Figure 16(a) shows that the $\beta$ ranges from $0^{\circ}$ to $180^{\circ}$ at the suction side. Taking the synergy angle of $90^{\circ}$ as the division, the clearance could be separated into two regions which contains the positive region and the negative region. Generally, the positive flow dominates the whole clearance, which indicates the leakage flow exists all along the flow direction. The negative region is mainly distributed in the upstream of the gap, where the SV appears. There is also a little part of negative region near the trailing edge, where the SB2 appears.
To validate the reliability of synergy-based method, the boundary (see the blue line), where the $w_{\theta}$ equals 0 , is employed to distinguish the positive region from the negative region, as shown in Figure 16(b). It could be concluded that the boundary of $\beta=90^{\circ}$ (see the red dotted line) agrees well with the numerical results especially near the trailing edge, where there exists the SB2. Nevertheless, the negative region has been overpredicted at the upper side of $\mathrm{CS}_{7}$. A possible explanation might be that the pressure difference near $\mathrm{CS}_{7}$ is so small (see Figure $9(\mathrm{~g})$ ) that the direction of pressure gradient might range from the suction side to pressure side partially. Thus, the leakage flow could be defined as the negative region in these places. The nondimensional leakage mass flow rate calculated by equation (8) is 0.0215

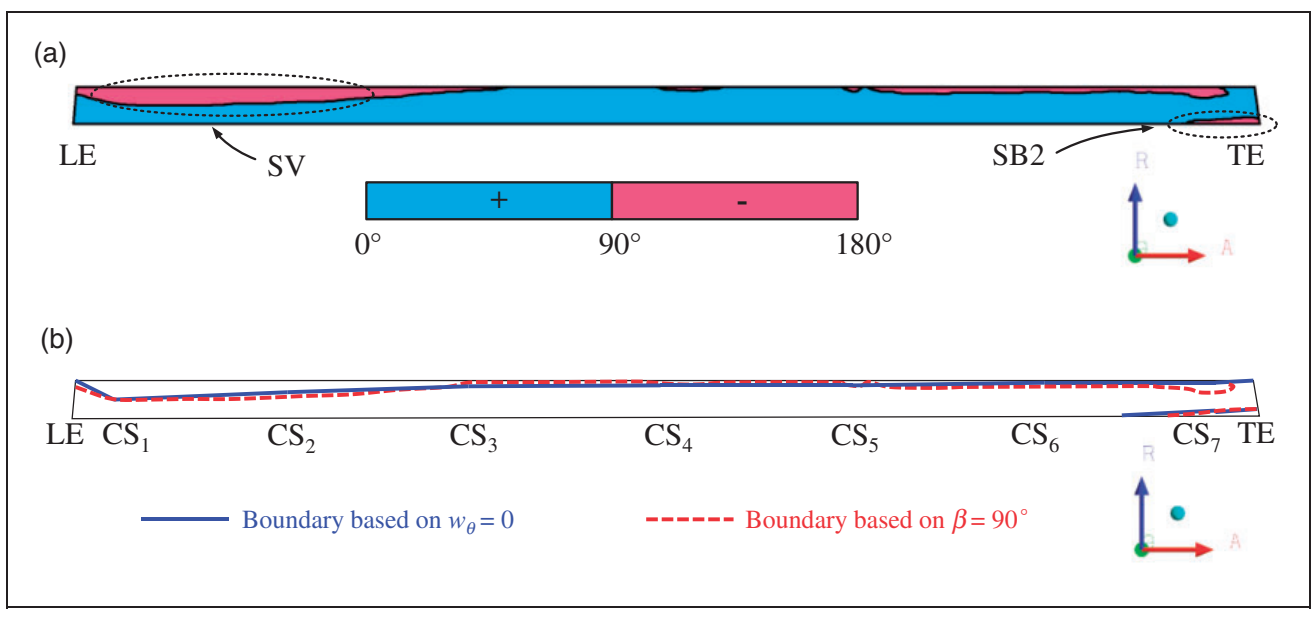

Figure 16. Distribution of the synergy angle at suction side of tip clearance: (a) boundary based on $90^{\circ}$ and (b) comparison of the boundary between the synergy method and $w_{\theta}=0$.

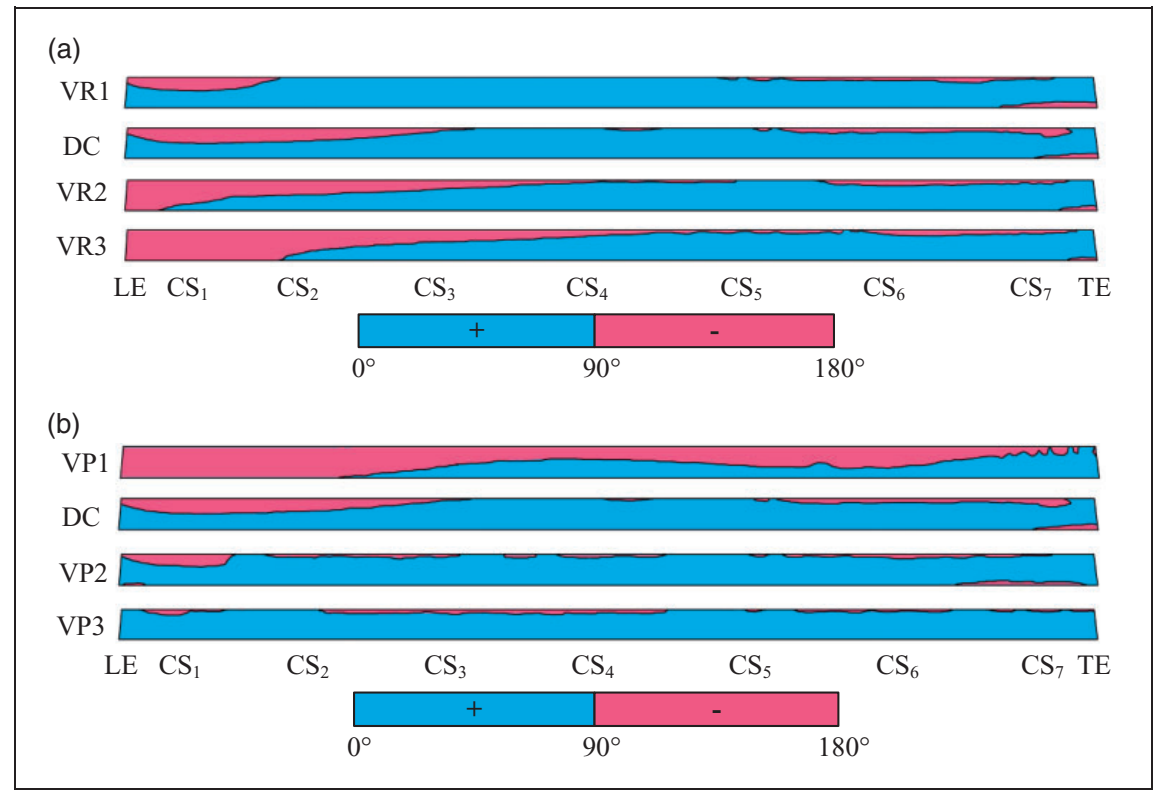

Figure 17. Schematic of the synergy angle at suction side of tip clearance under: (a) variable rotating speed conditions and (b) variable pressure ratio conditions. 


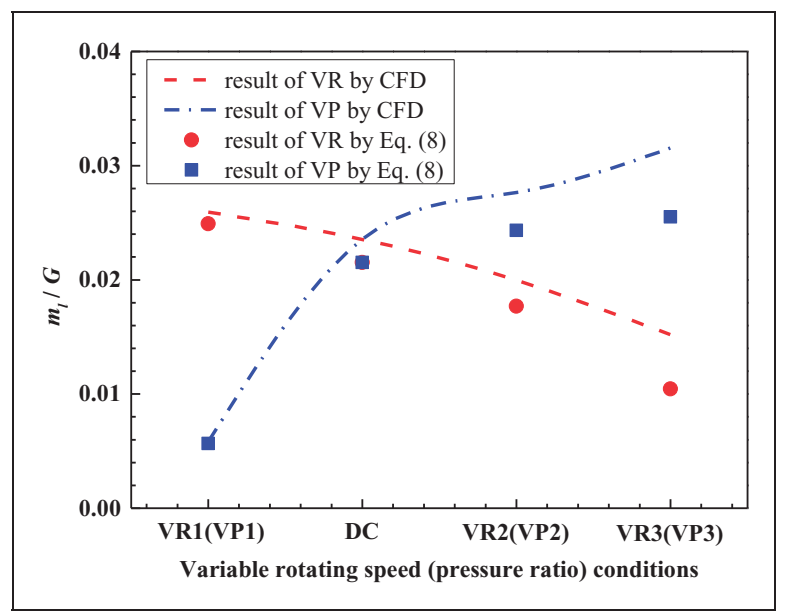

Figure 18. Nondimensional leakage mass flow rate at suction side of tip clearance under off-designed conditions.

while the numerical result is 0.0235 . The relative error of $-8.5 \%$ has been calculated.

Having examined the synergy relation and leakage mass flow rate at the designed condition, the results of flow structures at off-design conditions are displayed in Figure 17. When rotating speed rises, the negative region near the leading edge expands towards downstream significantly. The tip clearance is occupied by the scraping flow totally at the leading edge (e.g. VR2 or VR3), as shown in Figure 17(a). As the pressure ratio rises, the pressure difference $\Delta P$ through the tip gap also increases. Meanwhile, the leakage flow is enhanced and the area of positive region expands, as shown in Figure 17(b). These results suggest that the synergy angle could also present the flow patterns of tip clearance at off-designed conditions. Based on the flow patterns in Figure 17, the nondimensional leakage mass flow rate at off-design conditions is presented in Figure 18. The leakage mass flow rate is well estimated by equation (8) at low pressure ratio or low rotating speed conditions. For example, good agreements have been achieved at the VR1 and VP1 conditions, and their relative deviations are $-3.9 \%$ and $-2.6 \%$, respectively. However, the results at higher pressure ratio or higher rotating speed conditions show relatively larger deviations. A possible explanation for these results may be the flow pattern becomes more complicated at the clearance region. The direction of pressure gradient may be disturbed partially due to the aforementioned reasons. Overall, the relative root mean square errors are $16.2 \%$ and $17.9 \%$ at variable rotating speed and variable pressure ratio conditions, respectively. It is also worth noting that the synergy angle gives a reliable criterion of indicating the flow patterns in the tip clearance.

\section{Conclusions}

A numerical study has been conducted to examine the flow field at tip clearance of an axial-inflow turbine rotor at low Reynolds number conditions. The effects of static pressure on flow characteristics of tip clearance have been studied overall, and then the rules of scraping ratio and the leakage mass flow rate based on the synergy method have been discussed. Based on these investigations, a modified scraping ratio is brought forward. The synergy angle is also analyzed at off-designed conditions. The results can be summarized as follows:

1. The tip clearance flow has been greatly affected by the flow separation at low Reynolds conditions. The reattachment of the separation bubble is observed when the tip pressure rises and it is equal to the endwall pressure. When the tip pressure is lower than the endwall pressure, the reattachment vanishes but there's still a separation bubble occupying the whole blade tip. When the tip pressure on the suction side is higher than that on the pressure side, an open separation bubble appears at the blade tip near the trailing edge. It could be explained by the development of flow separation attached to the suction surface.

2. The distribution of scraping ratio at low Reynolds number is different from the previous studies. The contradiction especially occurs at the front part of the clearance. It is because a sharp increase of pressure difference contributes to the development of local inertial force which causes leakage flow. The modification shows satisfactory results at offdesign conditions, which considers the effect of low Reynolds number.

3. The synergy angle between velocity and pressure gradient gives a reliable method of researching the flow patterns in the tip clearance. Both the flow characteristics and leakage mass flow rate give satisfactory results compared to the CFD data at the designed operating condition. However, the leakage mass flow rate shows relatively larger deviations at higher pressure ratio and higher rotating speed conditions, whose flow pattern is far more complicated at the clearance region.

\section{Acknowledgements}

The authors would like to gratefully acknowledge Dr Takayuki Matsunuma of the National Institute of Advanced Industrial Science and Technology (AIST), for his kind supply of the blade geometry and experimental data.

\section{Declaration of conflicting interests}

The author(s) declared no potential conflicts of interest with respect to the research, authorship, and/or publication of this article.

\section{Funding}

The author(s) disclosed receipt of the following financial support for the research, authorship, and/or publication 
of this article: This work was supported by the National Key R\&D Plan through grant No. 2017YFB0903602, National Natural Science Foundation of China through grant No. 51522605, The frontier science research project of CAS through grant No. QYZDB-SSW-JSC023 and Newton Advanced Fellowship of the Royal Society through grant No. NA170093.

\section{ORCID iD}

Ziyi Shao (D) https://orcid.org/0000-0002-9330-4331

Xing Wang (D) https://orcid.org/0000-0002-4644-6599

Haisheng Chen (D) https://orcid.org/0000-0002-1383-9476

\section{References}

1. Bindon JP. The measurement and formation of tip clearance loss. J Turbomach 1989; 111: 257-263.

2. Moore $\mathbf{J}$ and Tilton JS. Tip leakage flow in a linear turbine cascade. J Turbomach 1988; 110: 18-26.

3. Heyes FJG and Hodson HP. Measurement and prediction of tip clearance flow in linear turbine cascades. J Turbomach 1993; 115: 376-382.

4. Yaras MI and Sjolander SA. Prediction of tip-leakage losses in axial turbines. J Turbomach 1992; 114: 204-210.

5. Denton JD. Loss mechanisms in turbomachines. J Turbomach 1993; 115: 621-656.

6. Booth TC. Importance of tip clearance flows in turbine design. VKI Lecture Series no. 1985-05, 1985.

7. Bunker RS. A review of turbine blade tip heat transfer. Ann NY Acad Sci 2006; 934: 64-79.

8. Zhou $\mathrm{C}$ and Hodson $\mathrm{H}$. The tip leakage flow of an unshrouded high pressure turbine blade with tip cooling. J Turbomach 2011; 133041028.1-041028.12.

9. Gao J, Fu WL, Wang FK, et al. Experimental and numerical investigations of tip clearance flow and loss in a variable geometry turbine cascade. Proc IMechE, Part A: J Power Energy 2018; 232: 157-169.

10. Zhou ZH, Chen SW and Wang ST. Aerodynamic optimisation of a winglet-cavity tip in a high-pressure axial turbine cascade. Proc IMechE, Part G: J Aerospace Engineering 2018; 232: 649-663.

11. Li J, Kong S, Yan X, et al. Numerical investigations on leakage performance of the rotating labyrinth honeycomb seal. J Eng Gas Turbines Power 2010; 132: 062501.1-062501.11.

12. Yan $\mathrm{X}, \mathrm{Li} \mathrm{J}$ and Feng Z. Effects of inlet preswirl and cell diameter and depth on honeycomb seal characteristics. J Eng Gas Turbines Power 2010; 132: 122506.1-122506.13.

13. Morphis $G$ and Bindon JP. The effects of relative motion, blade edge radius and gap size on the blade tip pressure distribution in an annular turbine cascade with clearance. ASME paper no. 88-GT-256, 1988.

14. Yaras MI and Sjolander SA. Effects of simulated rotation on tip leakage in a planar cascade of turbine blades: part I-tip gap flow. J Turbomach 1992; 114: 652-659.

15. Tallman $\mathbf{J}$ and Lakshminarayana B. Methods for desensitizing tip clearance effects in turbines. ASME paper no. 2001-GT-0486, 2001.

16. Cho SY, Cho CH and Choi SK. An experimental study of partial admission losses with various blade tip clearances using a linear cascade. Energy 2017; 122: 627-637.
17. Dixon SL and Hall C. Fluid mechanics and thermodynamics of turbomachinery. Oxford: ButterworthHeinemann, 2010.

18. Matsunuma T. Effects of Reynolds number and freestream turbulence on turbine tip clearance Flow. $J$ Turbomach 2006; 128: 166-177.

19. Rizzetta D and Visbal M. Numerical investigation of plasma-based flow control for a transitional highly-loaded low-pressure turbine. AIAA J 2007; 45: 2554-2564.

20. Dorney D, Lake J, King P, et al. Experimental and numerical investigation of losses in low-pressure turbine blade rows. In: 38th aerospace sciences meeting and exhibit, AIAA 2000-0737, 2000.

21. Arakawa H, Suzuki T, Saito K, et al. Research and development of $300 \mathrm{~kW}$ class ceramic gas turbine project in Japan. ASME paper no. 97-GT-87, 1997.

22. Matsunuma T. Unsteady flow field of an axial-flow turbine rotor at a low Reynolds number. $J$ Turbomach 2007; 129: 360-371.

23. Matsunuma T. Effects of nozzle secondary vortices on unsteady hub-endwall flow of a turbine rotor. In: ICOPE 2007, 2007, pp.331-337.

24. Dambach R, Hodson HP and Huntsman I. An experimental study of tip clearance flow in a radial inflow turbine. J Turbomach 1998; 121: 644-650.

25. Teuber R, Wilson M, Li YS, et al. Computational extrapolation of turbine sealing effectiveness from test rig to engine conditions. Proc IMechE, Part A: J Power Engineering 2012; 227: 167-178.

26. He P, Sun Z, Guo B, et al. Aerothermal investigation of backface clearance flow in deeply scalloped radial turbines. J Turbomach 2013; 135: 021002.1-021002.12.

27. Ainley DG and Mathieson GCR. A method of performance estimation for axial-flow turbines. Aeronautical Research Council Technical Report R\&M, No. 2974; 1951.

28. ICEM-CFD 15.0 theory guide. Canonsburg, PA: ANSYS Inc., 2013.

29. Menter FR. Two-equation eddy-viscosity turbulence models for engineering applications. AIAA J 1994; 32: 1598-1605.

30. Serrano JR, Navarro R, García-Cuevas LM, et al. Turbocharger turbine rotor tip leakage loss and mass flow model valid up to extreme off-design conditions with high blade to jet speed ratio. Energy 2018; 147: 1299-1310.

31. Liu W, Liu ZC and Guo ZY. Physical quantity synergy in laminar flow field of convective heat transfer and analysis of heat transfer enhancement. Chinese Sci Bull 2009; 54: 1779-1785.

32. Liu W, Liu ZC, Ming TZ, et al. Physical quantity synergy in laminar flow field and its application in heat transfer enhancement. Int J Heat Mass Transfer 2009; 52: 4669-4672.

33. Schlichting H and Gersten K. Boundary-layer theory. Berlin Heidelberg: Springer-Verlag, 2017.

34. Van Treuren KW, Simon T, von Koller M, et al. Measurements in a turbine cascade flow under ultra low Reynolds number conditions. ASME paper no. 2001-GT-0164, 2001.

35. Volino RJ. Control of tip leakage in a high pressure turbine cascade using tip blowing. ASME paper no. GT2016-56511, 2016. 
36. Sjolander SA and Cao D. Measurements of the flow in an idealized turbine tip gap. $J$ Turbomach 1995; 117: $578-584$.

37. Deng QH, Niu JF and Feng ZP. Study on leakage flow characteristics of radial inflow turbines at rotor tip clearance. Sci China Ser E: Technol Sci 2008; 51: 1125-1136.

38. Yaras M, Zhu YK and Sjolander SA. Flow field in the tip gap of a planar cascade of turbine blades. J Turbomach 1989; 111: 276-283.

\section{Appendix}

\section{Notation}

A

$c$

$C P S$

$C P t$

$\mathrm{CS}$

$\mathrm{DC}$

$\mathrm{G}$

\section{H}

$H_{12}$

LE

$m$

$\mathrm{Ma}$

PS

$P s$

$P t$

$R$

$\mathrm{Re}$

SB1

SB2

SBE

SS

SV

TC

TE

$T t$

$T u$ area $\left(\mathrm{m}^{2}\right)$

blade chord length $(\mathrm{mm})$

static pressure coefficient

total pressure loss coefficient

cross-sectional plane

designed condition

turbine mass flow rate $\left(\mathrm{kg} \mathrm{s}^{-1}\right)$

blade height $(\mathrm{mm})$

shape factor $\left(\delta_{1} / \delta_{2}\right)$

leading edge

mass flow rate $\left(\mathrm{kg} \mathrm{s}^{-1}\right)$

Mach number

pressure side

static pressure $(\mathrm{Pa})$

total pressure $(\mathrm{Pa})$

scraping ratio

Reynolds number

separation bubble

open separation bubble

separation bubble near the shroud

endwall

suction side

scraping vortex

tip clearance

railing edge

total temperature $(\mathrm{K})$

turbulence intensity
$U$

$u$

V

VP

VR

$\mathrm{W}$

Z

$\Delta P$

$\alpha$

$\beta$

$\gamma$

$\delta$

$\delta_{1}$

$\delta_{2}$

$\delta^{\prime}$

$\mu$

$\rho$

$\psi$

$\omega$

\section{Subscripts}

abs

in

$\mathrm{m}$

1

OB

out

r

RT

ST

tip

$\theta$

$-$

$+$

\section{Superscripts}

circumferential velocity $\mathrm{m} \mathrm{s}^{-1}$ mass-averaged velocity, $\mathrm{m} \mathrm{s}^{-1}$ absolute velocity, $\mathrm{m} \mathrm{s}^{-1}$ variable pressure ratio condition, Variable rotating speed condition, relative velocity, $\mathrm{m} \mathrm{s}^{-1}$

axial position of blade, -

Driving pressure difference, $\mathrm{Pa}$

Absolute flow angle, ${ }^{\circ}$

Synergy angle,

Local blade angle, ${ }^{\circ}$

Tip clearance, $\mathrm{mm}$

Displacement thickness, mm

Momentum thickness, $\mathrm{mm}$

Dimensionless tip clearance $(\delta / H)$, -

Dynamic viscosity, $\mathrm{Pa} \mathrm{s}$

Density, $\mathrm{kg} \mathrm{m}^{-3}$

Coefficient for modification, -

Rotating speed, rpm

Absolute flow or absolute frame

Inlet value of the domain

Modified value

Leakage flow

Outblock domain

Outlet value of the domain

Radial component

Rotor domain

Stator domain

Blade tip

Circumferential component

Negative mass flow direction identifier

Positive mass flow direction identifier 